\title{
An on-site dosimetry audit for high-energy electron beams
}

\author{
NEDERLANDSE COMMISSIE VOOR STRALINGSDOSIMETRIE
}

Report 29 of the Netherlands Commission on Radiation Dosimetry

October 2018 


\title{
An on-site dosimetry audit for high-energy electron beams
}

\author{
NEDERLANDSE COMMISSIE VOOR STRALINGSDOSIMETRIE
}

Report 29 of the Netherlands Commission on Radiation Dosimetry

\author{
Authors \\ Leon de Prez \\ Stan Heukelom \\ Bartel Jansen \\ Wim Jansen \\ Elfried Kok \\ Thijs Perik \\ Jacco de Pooter
}

Frits Wittkämper (Chairman) 


\section{Disclaimer regarding NCS reports}

The NCS frequently publishes reports for fellow professionals in which recommendations are given for various quality control procedures or otherwise. The members of the NCS board and the members of the concerning subcommittee do not claim any authority exceeding that of their professional expertise. Responsibility on how the NCS recommendations are implemented lies with the user, taking into account the practice in his/her institution.

Certain commercial equipment, instruments, or materials are identified in this report in order to specify the experimental procedure adequately. Such identification does not imply recommendation or endorsement, nor that the materials or equipment identified are necessarily the best available for the purpose. 


\section{Preface}

The Nederlandse Commissie voor Stralingsdosimetrie (NCS, Netherlands Commission on Radiation Dosimetry, http://www.radiationdosimetry.org) was officially established on 3 September 1982 with the aim of promoting the appropriate use of dosimetry of ionizing radiation both for scientific research and practical applications. The NCS is chaired by a board of scientists, installed upon the suggestion of the supporting societies, including the Nederlandse Vereniging voor Radiotherapie en Oncologie (Netherlands Society for Radiotherapy and Oncology), the Nederlandse Vereniging voor Nucleaire Geneeskunde (Dutch Society of Nuclear Medicine), the Nederlandse Vereniging voor Klinische Fysica (Dutch Society for Medical Physics), the Nederlandse Vereniging voor Radiobiologie (Netherlands Radiobiological Society), the Nederlandse Vereniging voor Stralingshygiëne (Netherlands Society for Radiological Protection), the Nederlandse Vereniging voor Medische Beeldvorming en Radiotherapie (Dutch Society for Medical Imaging and Radiotherapy), the Nederlandse Vereniging van Klinisch Fysisch Medewerkers (Dutch Society for Medical Physics Engineers), the Nederlandse Vereniging voor Radiologie (Radiological Society of the Netherlands) and the Belgische Vereniging voor Ziekenhuisfysici/Société Belge des Physiciens des Hôpitaux (Belgian Hospital Physicists Association). To pursue its aims, the NCS accomplishes the following tasks: participation in dosimetry standardisation and promotion of dosimetry intercomparisons, drafting of dosimetry protocols, collection and evaluation of physical data related to dosimetry. Furthermore, the commission shall maintain or establish links with national and international organisations concerned with ionizing radiation and promulgate information on new developments in the field of radiation dosimetry.

Current members of the board of the NCS

J.B. van de Kamer, Chairman T.W.M. Grimbergen, Vice-Chairman J.A. de Pooter, Secretary J.M.J. Hermans, Treasurer A. Rijnders N. De Graaf F.W. Wittkämper M.K. de Fluiter-Zeeman J.R. de Jong P. Sminia N.A.P. Franken 


\title{
An on-site dosimetry audit for high-energy electron beams
}

Members of the subcommittee:

\author{
Frits Wittkämper \\ Stan Heukelom \\ Bartel Jansen \\ Wim Jansen \\ Elfried Kok \\ Thijs Perik \\ Jacco de Pooter (Advisor) \\ Leon de Prez
}

NCS, Delft, the Netherlands

For more information on NCS Reports, see http://radiationdosimetry.org 


\section{Summary}

The NCS code of practice for high-energy photon and electron beams [1] has currently been implemented in most of the Dutch and Belgium radiotherapy institutes. Recently, the NCS performed a photon dosimetry audit at a large number of Dutch and Belgium radiotherapy departments [2] that has been continued as a VSL dosimetry service. In order to implement an electron audit rather quickly and efficiently, the NCS decided to establish a small audit group amongst four radiotherapy departments which already implemented the NCS 18 [1] code of practice for electron beams. The goal of this subcommittee was to establish a dosimetry audit for absorbed dose to water, $D_{\mathrm{w}}$, in electron beams.

The subject of the audit is the determination of absorbed dose to water, $D_{\mathrm{w}}$ in cGy, at reference depth, $z_{\text {ref, }}$ as well as the beam quality specifier, $R_{50 \text {,dos }}$ in $\mathrm{cm}$, in high-energy electron beams in the participant's accelerator facility as performed by local staff. The audit results are expressed as an $E_{n}$-score [3], where the outcome of the audit is either 'satisfactory' when $\left|E_{n}\right| \leq 1.0$ or 'unsatisfactory' when $\left|E_{n}\right|>1.0$.

The so called 'reference values' of $R_{50, \text { dos }}$ and $D_{\mathrm{w}}$ as measured by the audit team were performed according to NCS 18 [1]. Measurements of $D_{\mathrm{w}}$ and the percentage depth ionization curve (PDI) to obtain the beam quality, $R_{50, \text { dos }}$, were performed with a plane-parallel ionization chamber. $D_{\mathrm{w}}$ was based on a plane-parallel chamber's ${ }^{60} \mathrm{Co}$ calibration. Additionally, if an electron energy with $R_{50, \text { dos }}>7 \mathrm{~cm}$ was available, a cross-calibration of the plane-parallel chamber against a Farmer type chamber was performed.

The expanded uncertainty for $R_{50, \mathrm{dos}}$ was estimated to be $0.14 \mathrm{~cm} \quad(k=2)$. The relative expanded uncertainty in $D_{\mathrm{w}}$ at $z_{\text {ref }}$ with a plane-parallel chamber calibrated in ${ }^{60} \mathrm{Co}$ was estimated to be $3.6 \%(k=2)$.

For the uncertainty in the $D_{\mathrm{w}}$ audit result, $\Delta_{D_{\mathrm{w}}}$, correlations between the measured values by audit team and the participant were taken into account. This resulted in an estimated relative uncertainty for $\Delta_{D \mathrm{w}}$ of $2.4 \%$. The uncertainty in the audit result for $R_{50 \text {,dos }}$ was estimated to be $0.20 \mathrm{~cm}$.

A total of 15 electron beams were measured in 4 participating institutes. One institute was measured twice, i.e. at the beginning of the audit campaign as an initial test audit and at the end of the audit campaign as an actual audit. Both results have been reported.

All $R_{50 \text {,dos }}$ audit results, $\Delta_{R 50, \text { dos }}$ turned out to be satisfactory (i.e. $\left|E_{n}\right| \leq 1.0$ ) except for one electron beam with an unsatisfactory $E_{n}$-score of 1.4. It did not influence the audit result on the $D_{\mathrm{w}}$ measurement and therefore the overall audit result of this participant. All $D_{\mathrm{w}}$ audit results, $\Delta_{D \mathrm{w}}$, turned out to be satisfactory (i.e. $\left|E_{n}\right| \leq 1.0$ ). 
Overall it can be concluded that the audit was implemented successfully. Differences of $D_{\mathrm{w}}$ values between the audit team and the participant were in all cases smaller than $1 \%$ and in most cases smaller than $0.5 \%$ with a relative uncertainty of $2.4 \%(k=2)$. Differences in $R_{50 \text {,dos }}$ were in all cases smaller than $0.3 \mathrm{~cm}$ and in most cases smaller than $0.2 \mathrm{~cm}$ with an uncertainty of $0.20 \mathrm{~cm}$. Except for the first (test) audit, $D_{\mathrm{w}}$ values obtained with a planeparallel chamber calibrated in ${ }^{60} \mathrm{Co}$ and cross-calibrated in a high-energy electron crosscalibration agreed within $0.3 \%$.

It must be noted that the audit described in this study, has been performed at radiotherapy departments traceable to VSL by applying the same code of practice as the audit team. If this audit would be performed at departments not traceable to VSL or that these departments apply a different dosimetry protocol than the audit team, the uncertainties reported in this study do not apply.

This NCS subcommittee had its kick-off meeting at 2 February 2015. 


\section{Contents}

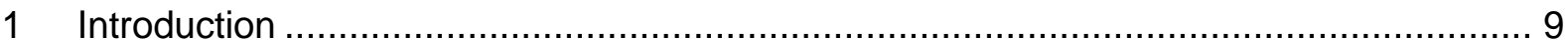

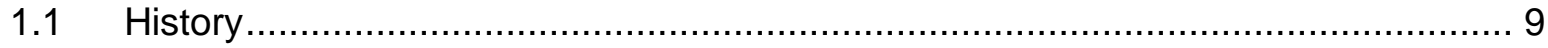

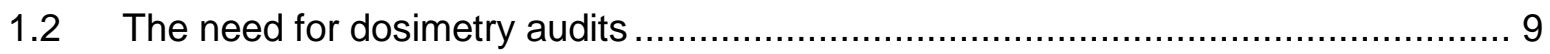

1.3 Goal of the NCS subcommittee 'Electron Audit' .............................................. 9



2.1 Subject and measured quantities ............................................................. 11

2.2 Reporting of the measurements and audit results ............................................11

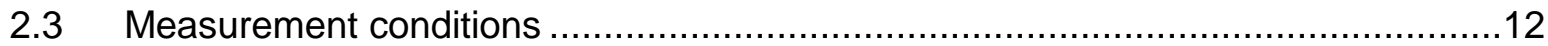

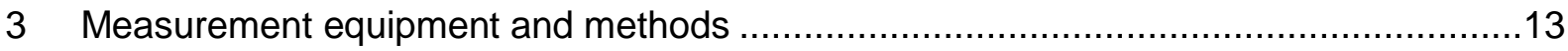

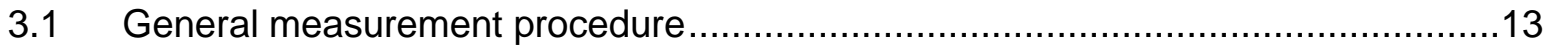

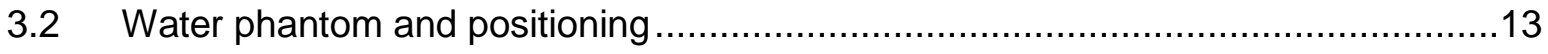

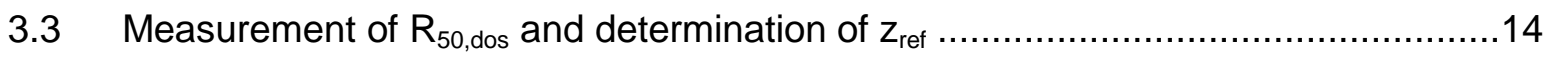

3.4 Measurement of percentage depth ionization curve, PDI ...................................14

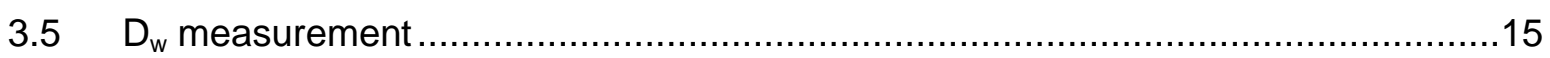

3.6 Calibration coefficient of the plane-parallel chamber in beam quality $Q, N_{D, w, Q}^{P P} \ldots \ldots 15$

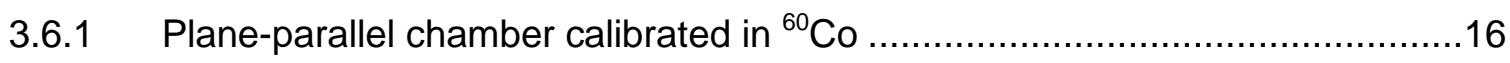

3.6.2 Plane-parallel chamber cross-calibrated in a high-energy electron beam ........16

3.7 Electrometer and ionization chamber correction factors .....................................17

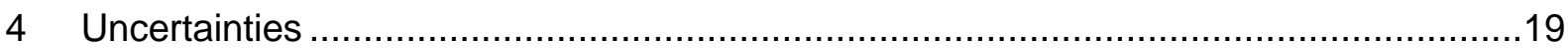

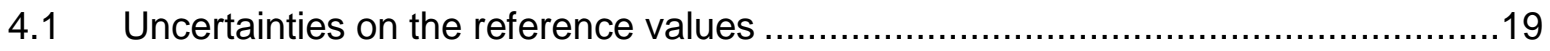



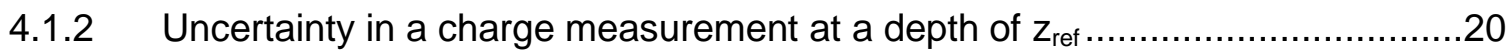

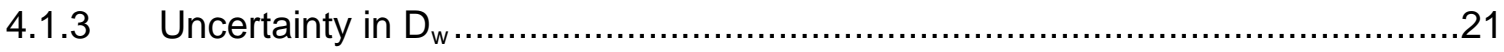

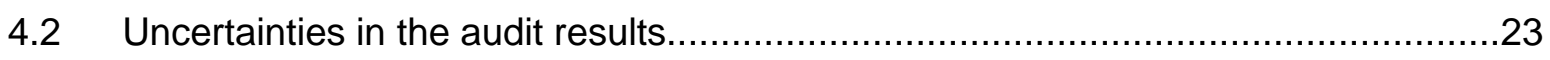

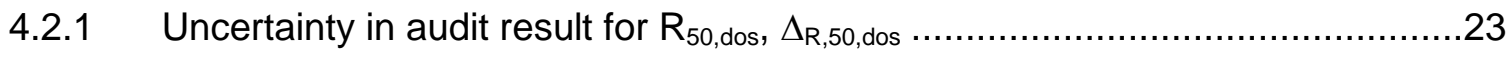

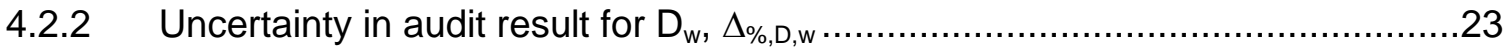

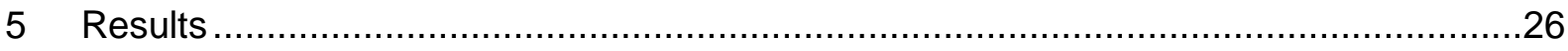

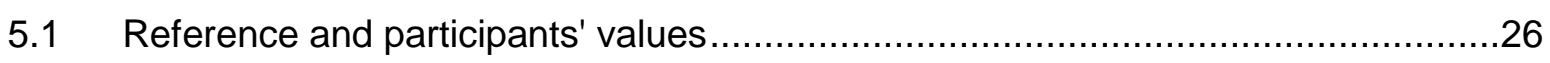

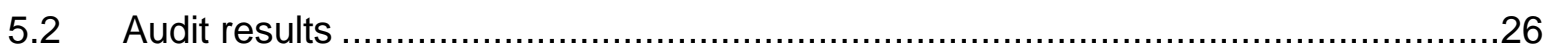

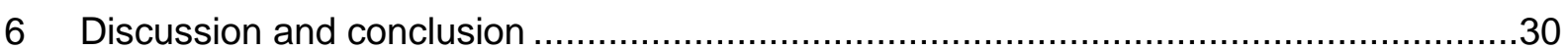

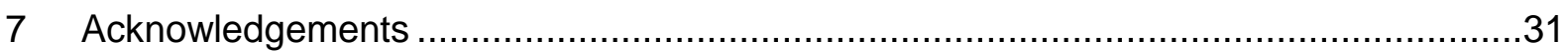






\section{Introduction}

\subsection{History}

In 2008 the Netherlands Commission on Radiation Dosimetry (NCS) issued a new code of practice for high-energy photon and electron beams based on absorbed dose to water standards, NCS 18 [1]. The new code of practice (CoP) replaced the air kerma based CoP described in NCS reports 2 and 5 [4,5]. NCS 18 is currently adopted or being implemented in the majority of the Dutch and Belgium radiotherapy departments.

\subsection{The need for dosimetry audits}

An external dosimetry audit after introducing a new dosimetry protocol or on a periodical basis is a powerful quality assurance instrument for radiotherapy departments. Therefore, the NCS decided to install a subcommittee with the aim to develop a reference dosimetry audit for electron beams to enable independent validation of the implementation of NCS 18 for electron beams. After development of the method and performing the audit, the audit method is transferred to VSL, the Dutch national metrology institute, which will offer audits for electron beams to users upon request. A similar approach was applied to set-up the photon beam audit, described in NCS report 23 [2], where the measurement protocol and procedures of the NCS subcommittee were adopted by VSL. This approach assures that the VSL adopted audit service is in accordance with the needs of radiotherapy departments.

To reduce workload and enhance efficiency of the subcommittee it was decided to perform the audit for a relatively small group of radiotherapy departments (Table 1.1), which already adopted the NCS 18 [1] code of practice for their electron dosimetry.

Table 1.1: Participating radiotherapy departments.

\begin{tabular}{l|lll}
\hline name & short & city & site location \\
\hline The Netherlands Cancer Institute & AVL & Amsterdam & Amsterdam \\
Leiden University Medical Center & LUMC & Leiden & Leiden \\
Reinier de Graaf & RdG & Delft & Delft \\
VU University Medical Center & VUmc & Amsterdam & Hoorn \\
\hline
\end{tabular}

\subsection{Goal of the NCS subcommittee 'Electron Audit'}

The goal of the NCS subcommittee 'Electron Audit' is to establish an electron dosimetry audit for absorbed dose to water, $D_{\mathrm{w}}$, and electron beam quality, $R_{50, \text { dos }}$, under defined reference conditions. Table 1.2 summarizes the electron beams chosen for testing the audit protocol 
and measurements. The audit results are based on the $R_{50, \text { dos }}$, and $D_{\mathrm{w}}$ at reference depth, $Z_{\text {ref- }}$

The visit at the first radiotherapy department was considered a 'test' audit in order to become familiar with the audit equipment in an electron dosimetry setting. The audit at this radiotherapy department was repeated at the end of the measurement campaign as the actual audit for this radiotherapy department.

Table 1.2: Overview of the electron beams. Here SSD is the Source Detector Distance and 'isoc' refers to the beam's iso-centre ${ }^{2}$.

\begin{tabular}{ccccc}
\hline participant & linear accelerator & nominal energies & SSD & field size at isoc \\
& type & /MeV & /cm & $/ \mathbf{c m}^{2}$ \\
AVL & Elekta Synergy (MLCi) & $6 ; 12 ; 18$ & 95 & $10.5 \times 10.5$ \\
RdG & Elekta Synergy (Agility) & $4 ; 10 ; 15$ & 100 & $10.5 \times 10.5$ \\
VUmc & Varian TrueBeam & $6 ; 9 ; 22$ & 100 & $10 \times 10$ \\
LUMC & Elekta Synergy (MLCi) & $4 ; 12 ; 4 \mathrm{HDRE}^{1}$ & 100 & $10.5 \times 10.5 / 42 \times 42^{1}$ \\
AVL & Elekta Synergy (MLCi) & $6 ; 12 ; 18$ & 95 & $10.5 \times 10.5$ \\
\hline
\end{tabular}

\footnotetext{
High Dose Rate Electron beam (HDRE) with field size of $42 \times 42 \mathrm{~cm}^{2}$, which is larger than the surface of the audit phantom.

2 The field size is determined by the accelerator applicator. For Elekta accelerators the field size is defined at 95 $\mathrm{cm}$, thus for a $10 \times 10 \mathrm{~cm}^{2}$ applicator the field size in the iso-centre is $10.5 \times 10.5 \mathrm{~cm}^{2}$. For Varian accelerators the field size is defined at $100 \mathrm{~cm}$, resulting in a field size in the iso-centre of $10 \times 10 \mathrm{~cm}^{2}$.
} 


\section{Audit protocol}

\subsection{Subject and measured quantities}

The subject of the audit is the determination of absorbed dose to water, $D_{w}$ in cGy, at reference depth, $z_{\text {ref, }}$, as well as the beam quality specifier, $R_{50 \text {,dos }}$ in $\mathrm{cm}$, in high-energy electron beams. The participant's staff performs measurements according to local dosimetry procedures and using local equipment. The NCS-team performs measurements according to the procedures described in this report and using the NCS-team's equipment. The NCS measurements are referred to as the audit's 'reference values'. Participant and NCS-team perform $D_{\mathrm{w}}$ and $R_{50, \text { dos }}$ measurements on the same day.

\subsection{Reporting of the measurements and audit results}

The participant's values and reference values are part of the audit report. It is unusual for radiotherapy departments to report uncertainties. NCS-team and participant perform their dosimetry at the same reference conditions and with similar, calibrated equipment. Therefore, it is unlikely that the participant's uncertainties are smaller than those of the NCSteam. For reasons of simplicity, it is assumed that the uncertainties on the participant's values are at least that of the uncertainty on the reference value.

The audit is established as a comparison between the measured value, $x$, by the participating institute and measured reference value, $X$, by the NCS audit team. The evaluation of the comparison is expressed as an, $E_{n}$-score [3]:

$$
E_{n}=\frac{\Delta}{U_{\Delta}}
$$

where $\Delta$ is the difference between the participant's value, $x$, and the reference value, $X$ :

$$
\Delta=X-X
$$

$x \quad$ is the measured value by the participant;

$X \quad$ is the assigned reference value, measured by the NCS audit team;

$U_{\Delta} \quad$ is the expanded uncertainty $(k=2)$ on the difference, $\Delta$.

The outcome of the audit, expressed as $E_{n}$, in accordance with [3], is either:

$$
\text { 'satisfactory' when }\left|E_{n}\right| \leq 1.0
$$

or

'unsatisfactory' when $\left|E_{n}\right|>1.0$ 
The audit result in the $R_{50, \text { dos }}$ (section 3.3) is expressed as an absolute value in the unit $\mathrm{cm}$ :

$$
\Delta_{R_{50, \text { dos }}}=R_{50, \text { dos }}-R_{50, \text { dos, ref }}
$$

The audit result in the $D_{\mathrm{w}}$ measurement (see section 3.5 ) is expressed as a relative value, expressed in:

$$
\Delta_{D_{w}}=\frac{D_{\mathrm{w}}-D_{\mathrm{w}, \text { ref }}}{D_{\mathrm{w}, \text { ref }}}
$$

Combining equations (1), (3) and (4), the audit results are evaluated using a normalized error, $E_{n}$-score:

$$
\mathrm{E}_{\mathrm{n}, R_{50, \mathrm{dos}}}=\frac{\Delta_{R_{50, \mathrm{dos}}}}{U_{\Delta_{R_{50, \mathrm{dos}}}}}
$$

and

$$
\mathrm{E}_{\mathrm{n}, D_{\mathrm{w}}}=\frac{\Delta_{D_{\mathrm{w}}}}{U_{\Delta_{D_{\mathrm{w}}}}}
$$

where, $U_{\Delta_{R_{50, \mathrm{dos}}}}$, in $\mathrm{cm}$, is the expanded uncertainty for the audit result in $R_{50, \mathrm{dos}} . U_{\Delta_{D_{\mathrm{w}}}}$, in \%, is the expanded relative uncertainty on the audit result for $D_{\mathrm{w}}$. In this study the overall outcome of the audit is only based on the $\mathrm{E}_{\mathrm{n}}$-score for the $D_{\mathrm{w}}$ measurement.

\subsection{Measurement conditions}

The reference measurements are carried out according to NCS 18 [1]. The reference measurements by the NCS audit team are performed at the same SSD (Source Surface Distance) as the participant's measurements. The $D_{\mathrm{w}}$ measurements performed by both the NCS audit team and the participant are performed at the same number of accelerator monitor units. The following reference conditions apply:

- source surface distance, SSD: $95 \mathrm{~cm}$ or $100 \mathrm{~cm}$ (depending on participant's SSD);

- depth in water, $z_{\text {ref: }}$ based on $R_{50, \text { dos }}$ according to equation (8) in section 3.3;

- Field size at iso-center: $\geq 10 \times 10 \mathrm{~cm}^{2}$, applied according to Table 1.2;

- Water phantom: $\geq 30 \times 30 \times 30 \mathrm{~cm}^{3}$ (see section 3.2).

The required ambient conditions for the measurements are:

- phantom (water) temperature, $T_{\mathrm{w}}: 16^{\circ} \mathrm{C}<T_{\mathrm{w}}<26^{\circ} \mathrm{C}$;

- room temperature, $T_{\text {room }}:\left(T_{\mathrm{w}}-4^{\circ} \mathrm{C}\right)<T_{\text {room }}<\left(T_{\mathrm{w}}+4^{\circ} \mathrm{C}\right)$;

- ambient pressure, $p$ : atmospheric;

- relative humidity, $R H \%: 20 \%-80 \%$. 


\section{Measurement equipment and methods}

\subsection{General measurement procedure}

The general audit procedure is as follows. After setting up the audit equipment and aligning the water phantom, as described in section 3.2, percentage depth ionization (PDI) curves and $D_{\mathrm{w}}$ are measured, as described in sections 3.3 and 3.5 respectively. For practical reasons it was decided to use the same plane-parallel chamber for both the $D_{\mathrm{w}}$ and the PDI measurements. The $D_{\mathrm{w}}$ measurement with the plane-parallel chamber is traceable to primary standards via the chamber's ${ }^{60} \mathrm{Co}$ calibration. If an electron beam with beam quality $R_{50 \text {,dos }}>7 \mathrm{~cm}$ is part of the audit, a cross-calibration of the plan-parallel against a Farmertype chamber, as described in section 3.6.2, is done immediately following the $D_{\mathrm{w}}$ measurements in the highest energy. After the NCS-team performs the audit measurements, the participant is given time to perform their absolute dosimetry for the same electron energies. On the day of the audit only $D_{\mathrm{w}}$ is measured by the participant. However, in this study some participants also measured $R_{50, \text { dos }}$. The audit is finalized by a repetition of $D_{\mathrm{w}}$ measurement by the NCS-team. The complete electron audit measurement takes between 4 and 6 hours.

\subsection{Water phantom and positioning}

A water phantom with outer dimensions of $32 \times 37 \times 32 \mathrm{~cm}^{3}(L \times W \times H)$ and wall thickness of $2 \mathrm{~cm}$ is used by NCS-team (PTW MP1 T41025). The phantom tank is equipped with an automated vertical translation stage and control unit (PTW TBA). A custom made PMMA (polymethyl methacrylate) bridge is used to mount ionization chambers on the translation stage's vertical carriage by means of the PTW mounting system. The purpose of the bridge is to place the chamber in the centre of the phantom and to minimize beam scatter as a result of the beam hitting metal parts of the translation stage.

The water phantom is placed on top of the patient couch. The couch is used to move the phantom up and down as desired. A calibrated metal ruler attached to the side of the water phantom is used to adjust the patient treatment couch' displacement in vertical direction in relation to the room laser in a similar way as described by Perik et al. [2]. Note that the displacement indicator of the couch is not used in order to avoid possible displacement errors. 


\subsection{Measurement of $R_{50, \text { dos }}$ and determination of $z_{\text {ref }}$}

$R_{50 \text {,dos }}$ is determined by means of the measured percentage dose ionization (PDI) curves, described in section 3.4. $R_{50 \text {,dos }}$ is based on the distance between the water surface and the point beyond the dose maximum, where the PDI has a value of $50 \%, R_{50 \text {,ion }}$ according to [1]:

$$
\begin{array}{ll}
R_{50, \text { dos }} & =1.029 R_{50, \text { ion }}-0.06 \mathrm{~cm} \quad \text { for } R_{50 \text {,ion }} \leq 10 \mathrm{~cm} \\
R_{50, \text { dos }}=1.059 & R_{50 \text {,ion }}-0.37 \mathrm{~cm} \quad \text { for } R_{50, \text { ion }}>10 \mathrm{~cm}
\end{array}
$$

Additionally, the $R_{50 \text {,dos }}$ is determined from the percentage depth dose curve (PDD) after conversion of the PDI to a PDD. This is done by multiplying the PDI with the mass stopping power ratios $\mathrm{s}_{\mathrm{w}, \text { air }}$ according to the method described by Andreo et al. [6]. In this study, $R_{50, \text { dos }}$ determined with both methods (based on PDI conversion and based applied in according to equation (7) have been compared. Differences were always smaller than $0.03 \mathrm{~cm}$ and therefor considered insignificant. $R_{50 \text {,dos }}$ is used to determine the reference measurement depth for the $D_{\mathrm{w}}$ measurements, $z_{\mathrm{ref}}$, and determined according to [1]:

$$
z_{\text {ref }}=0.6 R_{50, \text { dos }}-0.1 \mathrm{~cm}
$$

\subsection{Measurement of percentage depth ionization curve, PDI}

The PDI is measured with the plane-parallel ionization chamber based on the position of its effective point of measurement (EPOM), for the used plane-parallel ionization chamber the EPOM is $0.112 \mathrm{~cm}$ behind the chambers' front surface. NCS 18 [1] recommends to use a $20 \times 20 \mathrm{~cm}^{2}$ field at the high-energy electron beams $\left(R_{50, \text { dos }}>7 \mathrm{~cm}\right)$ because of possible insufficient scatter equilibrium at the central axis [7]. However, all PDI measurements are performed with a beam size close to $10 \times 10 \mathrm{~cm}^{2}$ (except for the 4HDRE beam at LUMC). The reason for this is that the applicator tray, with the monitor ionization chamber attached to it, doesn't need to be changed during the measurements of the PDI curves, i.e. the curves can be measured without interruption. In order to verify the validity of this method, PDI curves were measured in the highest energy beam (22 $\mathrm{MeV}$ at VUmc) both with beam sizes of $10 \times 10 \mathrm{~cm}^{2}$ and $20 \times 20 \mathrm{~cm}^{2}$. The measured PDI curves were converted to $R_{50 \text {,dos }}$ using equation (7). In this study the difference between $R_{50 \text {,dos }}$ in a $10 \times 10 \mathrm{~cm}^{2}$ field and in a $20 \times 20 \mathrm{~cm}^{2}$ field was found to be smaller than $0.04 \mathrm{~cm}$ and therefore considered negligible for beams up to $22 \mathrm{MeV}$.

Corrections for chamber polarity, $k_{\text {pol }}$, and irradiation of the stem, $k_{\text {stem }}$, with depth have not been applied. With respect to $k_{\mathrm{pol}}$, it is assumed that it's variation with depth, i.e. between $R_{100 \text {,ion }}$ and $R_{50 \text {,ion }}$ is negligible [8]. Additionally, the variation in stem effect with depth is considered to be insignificant and close to the PDD $50 \%$ point for all energies. 
Recombination varies as a function of depth, due to a varying dose per pulse. Therefore, the measured charge is corrected for recombination as a function of depth. This is done by the assumption that the recombination effect, determined at $z_{\text {ref }}$ with the two-voltage method recommended by NCS 18 [1], is dominated by volume recombination (neglecting initial recombination) and thus proportional to the depth dose relative to $D_{\mathrm{w}}$ at $z_{\text {ref }}$.

The NCS 18 [1] formalism is used to convert the measured $R_{50 \text {,ion }}$ value, uncorrected for recombination, to $z_{\text {ref }}$ at which $D_{\mathrm{w}}$ is measured. The recombination correction is subsequently measured at $z_{\text {ref }}$ according to the method described by Weinhous and Meli [9], by reducing the chamber voltage by a factor 4 . Hence, the reference depth, $z_{\text {ref }}$ at which the actual $D_{\mathrm{w}}$ measurement takes place is determined with $R_{50 \text {,ion }}$ uncorrected for $k_{\mathrm{s}}$. This causes a small error of up to $0.01 \mathrm{~cm}$ in the actual depth of the chamber in water compared to the desired $z_{\text {ref }}$ and has been accounted for in the uncertainty.

For the measurement of the PDIs an independent monitor ionization chamber (Semiflex PTW 31013 ) is used to normalize for beam output fluctuations. This monitor chamber is mounted on the applicator tray at the edge of the beam. Its sensitive volume is completely inside the electron beam but positioned such that it doesn't influence the reading of the detector. The plane-parallel and monitor ionization chambers are connected to a dual channel electrometer and are read out simultaneously while the translation stage is moved in vertical direction upwards. Data-acquisition of PDI is performed automatically by means of commercially available software (PTW MEPHYSTO $\mathrm{mc}^{2}$ tbaScan) and the obtained data is analysed in a spreadsheet.

\section{5 $D_{w}$ measurement}

The absorbed dose to water, $D_{\mathrm{w}}$, in electron beam quality, $Q$, is measured using a waterproof plane-parallel ionization (PTW 34001) chamber connected to a single channel electrometer (PTW UNIDOS T10002) and obtained according to the formalism described in NCS 18 [1]:

$$
D_{\mathrm{w}, Q}=M_{\text {corr }}^{P P} N_{D, \mathrm{w}, \mathrm{Q}}^{P P}
$$

where $M_{c o r r}^{P P}$ is the corrected electrometer reading (section 3.7) and $N_{D, \mathrm{w}, Q}^{P P}$ is the planeparallel chamber calibration coefficient in beam quality $Q$ (section 3.6).

\subsection{Calibration coefficient of the plane-parallel chamber in beam quality $Q, N_{D, \mathrm{w}, Q}^{P P}$}

The plane-parallel chamber's calibration coefficient in beam quality $Q, N_{D, w, Q}^{P P}$, is based on a ${ }^{60}$ Co calibration, $N_{D, w, Q 0}$, with beam quality correction $k_{Q, Q_{0}}^{P P}$. Additionally, a cross-calibration 
at a high-energy electron beam with $R_{50, \mathrm{dos}}>7 \mathrm{~cm}$ (when available) against a cylindrical chamber is performed. For the audit result the chamber's ${ }^{60} \mathrm{Co}$ calibration is used.

\subsubsection{Plane-parallel chamber calibrated in ${ }^{60} \mathrm{Co}$}

The plane-parallel chamber is calibrated in ${ }^{60} \mathrm{Co}$ with a beam size of $10 \times 10 \mathrm{~cm}^{2}$ at a Source Detector Distance $(S D D)$ of $100 \mathrm{~cm}$. The calibration coefficient, $N_{D, \mathrm{w}, Q}^{P P}$, in electron beam quality $Q$ is determined according to:

$$
N_{D, \mathrm{w}, Q}^{P P}=N_{D, \mathrm{w}, Q_{0}}^{P P} k_{Q, Q_{0}}^{P P}
$$

where $N_{D, w, Q_{0}}^{P P}$ is the ${ }^{60} \mathrm{Co}$ calibration of the plane-parallel ionization chamber and $k_{Q, Q_{0}}^{P P}$ is the plane-parallel chamber's beam quality dependent ionization chamber correction factor according to equation (11) and Table 2 in NCS 18 [1].

\subsubsection{Plane-parallel chamber cross-calibrated in a high-energy electron beam}

Optionally, a cross-calibration of the plane-parallel chamber against a Farmer type cylindrical chamber (NE2571) takes place in a high-energy electron beam with beam quality $R_{50 \text {,dos }}>7$ $\mathrm{cm}$ in the accelerator of the participant (when available). The cross-calibration procedure is followed according to NCS 18 [1] and $N_{D, w, Q}^{P P}$ in beam quality $Q$ is determined according to:

$$
N_{D, \mathrm{w}, Q}^{P P}=N_{D, \mathrm{w}, Q_{C R O S S}}^{P P} k_{Q, Q_{C R O S S}}^{P P}
$$

where $N_{D, w, Q}^{P P}$ is the plane-parallel chamber's cross-calibration coefficient in a high-energy electron beam with $R_{50 \text {,dos }}>7 \mathrm{~cm} . k_{Q, Q_{\text {cross }}}^{P P}$ is the ratio of beam quality correction factors for the plane-parallel chamber at beam quality $Q$ and at the cross-calibration quality $Q_{\text {cross, }}$ obtained from NCS 18 [1]. The plane-parallel chamber's calibration coefficient in $Q_{\text {cross, }}$ $N_{D, \mathrm{w}, Q_{C R O S S}}^{P P}$, is determined according to:

$$
N_{D, \mathrm{w}, Q_{C R O S S}}^{P P}=\frac{M_{Q_{C R O S S}}^{C Y L}}{M_{Q_{C R O S S}}^{P P}} N_{D, \mathrm{w}, Q_{0}}^{C Y L} k_{Q_{\text {cross }}^{C Y L} Q_{0}}^{C L}
$$

Here, $N_{D, w, Q_{0}}^{C Y L}$ is the ${ }^{60}$ Co calibration coefficient of the Farmer type cylindrical ionization chamber and $k_{Q, Q_{C R O S S}}^{C Y L}$ is the cylindrical chamber's beam quality correction factor according to equation (14) and Table 2 in NCS 18 [1]. $M_{Q_{C \text { ROSS }}}^{C Y L}$ and $M_{Q_{C \text { ROSS }}}^{P P}$ are the corrected electrometer 
readings, later indicated as $M_{\text {corr }}$ in equation (13), with the respective chambers positioned at $z_{\text {ref }}$ for a specified number of accelerator monitor units.

The plane-parallel and the cylindrical ionization chamber are subsequently connected to a single channel electrometer (PTW UNIDOS T10002), relying on the short-term stability of the accelerator monitor. The cylindrical ionization chamber is placed inside a VSL made waterproof PMMA sleeve with a wall thickness of $1 \mathrm{~mm}$. A thin latex tube is impermeably connected to the waterproof sleeve in order to keep the cylindrical ionization chamber and its cable dry when submerged.

During the cross-calibration procedure both ionization chambers are positioned with their effective point of measurement (EPOM) at a depth of $z_{\text {ref }}$ at the central axis of the beam. For the PTW Roos (PTW 34001) plane-parallel chamber the EPOM this is $0.112 \mathrm{~cm}$ from the front surface of the ionization chamber according to the manufacturer's documentation. For the cylindrical chamber the EPOM is at $0.5 \times$ the radius of the chamber central axis (i.e. $+0.158 \mathrm{~cm}$ for the NE2571 ionization chamber used in this study), also explained and illustrated in Figure 3 in NCS 18 [1]. This means that the centre of the cylindrical chamber is positioned $0.158 \mathrm{~cm}$ of the geometrical centre away from the accelerator focus. The bias voltages of the plane-parallel and cylindrical chamber are set to respectively $+200 \mathrm{~V}$ and $+300 \mathrm{~V}$.

\subsection{Electrometer and ionization chamber correction factors}

The corrected electrometer reading for the plane-parallel and the Farmer type cylindrical chamber, $M_{\text {corr }}$ (to improve readability the subscript 'corr' was omitted in the previous sections), is obtained according to:

$$
M_{\text {corr }}=M k_{\text {elec }} k_{p T} k_{\mathrm{s}} k_{\text {pol }}^{+}
$$

where the electrometer reading, $M$, is corrected for:

- electrometer calibration, $k_{\text {elec }}$ (electrometer and ionization chamber are calibrated as separate instruments, i.e. not as a dosimetry system);

- air cavity density compared to reference air density based on the ambient pressure and temperature, $k_{p}$;

- incomplete charge collection due to recombination, $k_{\mathrm{s}}[9]$;

- chamber polarity, $\mathrm{kol}^{+}{ }^{+}+$indicates an applied positive bias voltage).

All correction factors are determined according to generally accepted methods such as described in NCS 18 [1].

A correction for air cavity density, $k_{p T}$, compared to reference air density $\left(20{ }^{\circ} \mathrm{C}\right.$ and atmospheric pressure of $101.325 \mathrm{kP}$ ) is applied since the used ionization chambers are open 
to ambient air. The water temperature, used for $k_{p T}$, is measured with a thermometer (Omega $\mathrm{HH} 41$ ) with its thermistor positioned inside a stainless-steel waterproof sleeve at the same depth as the ionization chamber just outside the radiation field. The atmospheric pressure is determined inside the accelerator room (Vaisala PTU300).

Since the chamber's ${ }^{60} \mathrm{Co}$ calibration coefficients were corrected for polarity effect, in the electron beams a correction for chamber polarity, $\mathrm{kpol}^{+}$is applied based on a chamber positive bias voltage.

The ambient temperature and relative humidity are measured inside the accelerator room (Vaisala PTU300) in order to verify that the air temperature is not outside it's desired range. A correction for air relative humidity, $k_{h}$, is not applied since the chamber calibration coefficients have been measured at a relative humidity in the range between $20 \%$ and $80 \%$ for which the ionization chamber response varies $<0.1 \%[10]$.

Furthermore, no correction is applied for dose averaging over the chamber volume caused by beam radial non-uniformity, $k_{\mathrm{rn}}$, as it is a small effect regarding electron beam profiles used in this study. 


\section{Uncertainties}

\subsection{Uncertainties on the reference values}

The reported expanded uncertainty, $U$, on the measured reference values is based on the standard uncertainty, $u$, multiplied by a coverage factor $k=2$, which for a normal distribution corresponds to a coverage probability of approximately $95 \%$, unless mentioned otherwise. The standard uncertainty has been determined in accordance with the GUM 'Evaluation of measurement data - Guide to the Expression of Uncertainty in Measurement' [11]. Uncertainties are expressed in 2 significant digits with a minimum resolution of $0.01 \%$ or $0.01 \mathrm{~cm}$.

\subsubsection{Uncertainty in $R_{50, \text { dos }}$ and $z_{\text {ref }}$}

Table 4.1 gives the uncertainty budget for determination of $R_{50 \text {,dos }}$ in $\mathrm{cm}$. Where needed, relative uncertainty contributions due to charge measurements have been converted to $\mathrm{cm}$ with a sensitivity coefficient of $0.03 \mathrm{~cm} \%^{-1}$ based on a minimum PDD of $-4 \% \mathrm{~mm}^{-1}$ at $R_{50 \text {,dos }}$ at $22 \mathrm{MeV}$. All uncertainty contributions are of type B and are applicable for the percentage depth ionization curves (PDIs) described in section 3.4. The uncertainty budget for $R_{50 \text {,dos }}$ is based on a PDI measured with a Roos ionization chamber. Uncertainty contributions are added with respect to the conversion from PDI to PDD based on a variation of stopping power ratios, $s_{\mathrm{w}, \text { arr }}$, and positioning of the chamber. The standard uncertainty in determination of $R_{50 \text {,dos }}$ is estimated to be $0.07 \mathrm{~cm}$ (quadratic sum of two $0.05 \mathrm{~cm}$ uncertainties). The associated standard uncertainty in $z_{\text {ref }}$ due to $R_{50 \text {,dos }}$ is based on the uncertainty of $R_{50 \text {,dos }}$ and its conversion with a corresponding sensitivity coefficient of 0.6 estimated to be $0.04 \mathrm{~cm}$.

Table 4.1: Uncertainty budget for determination of $R_{50, \text { dos }}$ in $\mathrm{cm}$. All uncertainty contributions are of type B. Uncertainties in charge measurements are converted to depth with a sensitivity coefficient of $0.03 \mathrm{~cm} \%{ }^{-1}$.

\begin{tabular}{|c|c|}
\hline source of uncertainty & $\begin{array}{c}\text { standard } \\
\text { uncertainty } \\
\text { /cm }\end{array}$ \\
\hline alignment and positioning of plane-parallel chamber & 0.05 \\
\hline calibration of vertical translation stage & 0.04 \\
\hline beam energy change between measurement and audit (max. $1 \%$ output at $R_{50, \text { dos }}$ ) & 0.03 \\
\hline ratio of charge measurements at $\mathrm{PDI}_{\max }$ and at $\mathrm{PDI}_{50 \%}(u=0.1 \%)$ & 0.003 \\
\hline depth dependent correction for recombination, $k_{\mathrm{s}}(u<0.1 \%)$ & 0.003 \\
\hline depth dependent correction for chamber polarity, $k_{\text {pol }}{ }^{+}(u<0.1 \%)$ & 0.003 \\
\hline variation of $T$ and $p$ during a PDI measurement, $k_{p T}(u<0.2 \%)$ & 0.006 \\
\hline PDI to PDD conversion [6] $(u=0.2 \%)$ & 0.006 \\
\hline combined standard uncertainty, $u(k=1)$ & 0.07 \\
\hline expanded uncertainty, $U(k=2)$ & 0.14 \\
\hline
\end{tabular}




\subsubsection{Uncertainty in a charge measurement at a depth of $z_{\text {ref }}$}

Table 4.2 gives the uncertainty budget for the charge measurement procedure, including positioning of an ionization chamber. This uncertainty is used for the $D_{\mathrm{w}}$ measurement and for the cross-calibration of ionization chambers that both take place at a depth of $z_{\text {ref. }}$ The uncertainty in $S D D$ is based on (re-)positioning of the water phantom at the desired $S S D$ of $100 \mathrm{~cm}$ with an estimated standard uncertainty of $0.10 \mathrm{~cm}$. The uncertainly contribution due to the depth is based on a maximum dose gradient of $-0.4 \% \mathrm{~mm}^{-1}$ for $22 \mathrm{MeV}$ at $z_{\text {ref. }}$ The uncertainty in determination of $z_{\text {ref }}$ based on a measured $R_{50 \text {,dos }}$ (see Table 4.1 ) is $0.04 \mathrm{~cm}$ and taken into account here. Furthermore, the electrometer calibration, long-term drift and display resolution are taken into account with a combined uncertainty of $0.07 \%$. Uncertainties for the correction of cavity air density are dominated by the thermometer calibration (standard uncertainty, $u=0.05^{\circ} \mathrm{C}$ ) and the estimated temperature gradient between the ionization chamber and the point where the temperature is measured $\left(u=0.10^{\circ} \mathrm{C}\right)$ at a nominal water temperature of $20^{\circ} \mathrm{C}$, resulting in a relative standard uncertainly contribution of $0.04 \%$.

Table 4.2: Uncertainty for the determination of a corrected electrometer reading, $M_{\text {corr }}$ with an ionization chamber at a depth of $Z_{\text {ref, }}$ applicable for both plane-parallel and Farmer type cylindrical chambers. Uncertainties in positioning depth are converted to dose with a sensitivity coefficient of $-0.4 \% \mathrm{~mm}^{-1}$.

\begin{tabular}{lc}
\hline source of uncertainty & $\begin{array}{c}\text { standard } \\
\text { uncertainty /\% }\end{array}$ \\
\hline repeated charge measurement $($ type $\mathrm{A})$ & 0.10 \\
$S S D(u=0.1 \mathrm{~cm}$ at an $S D D$ of $100 \mathrm{~cm})$ & 0.20 \\
positioning of ionization chamber in water at $z_{\text {ref }}(u=0.05 \mathrm{~cm})$ & 0.20 \\
electrometer calibration, long term drift and resolution & 0.07 \\
$k_{p}:$ correction for ambient temperature and pressure & 0.10 \\
$k_{\mathrm{h}}(20 \%$ - $80 \%)$ : variation of relative humidity & 0.05 \\
$k_{\mathrm{s}}:$ correction for ion recombination & 0.10 \\
$k_{\text {pol }}{ }^{+}:$polarity correction & 0.10 \\
$k_{\mathrm{rn}}:$ variation due to beam radial non-uniformity & 0.10 \\
\hline combined standard uncertainty $(k=1)$ & 0.37 \\
expanded uncertainty, $U(k=2)$ & 0.74 \\
\hline
\end{tabular}




\subsubsection{Uncertainty in $D_{w}$}

Table 4.3 gives the uncertainty budget for a $D_{\mathrm{w}}$ measurement with a plane-parallel Roos chamber calibrated in ${ }^{60} \mathrm{Co}$. The combined standard uncertainty takes into account the contribution of the corrected electrometer reading, $M_{\text {corr }}$, based on the used measurement procedure (Table 4.2), the uncertainty of the ionization chamber calibration in ${ }^{60} \mathrm{Co}$ and the standard uncertainty in $k_{Q, Q 0}$ for the plane-parallel chamber [1]. The uncertainty contribution in $R_{50 \text {,dos }}$ as a result of the conversion from PDI to PDD using stopping power ratios is incorporated in the uncertainty of $k_{\mathrm{Q}, \mathrm{Q} 0}$ (see e.g. NCS 18 [1] and IAEA TRS-398 [6]).

Table 4.4 gives the uncertainty budget for $D_{w}$ in a high-energy electron beam with quality with $R_{50, \text { dos }}>7 \mathrm{~cm}$ measured with a Farmer type cylindrical ionization chamber calibrated in ${ }^{60} \mathrm{Co}$. The combined standard uncertainty takes into account the contribution on $M_{\text {corr }}$ (Table 4.2), the ${ }^{60} \mathrm{Co}$ calibration and the uncertainty in $k_{Q, Q 0}$.

Table 4.5 gives the uncertainty budget for a $D_{\mathrm{w}}$ measurement with a plane-parallel chamber cross-calibrated against a cylindrical chamber in a beam quality $Q$ with $R_{50 \text {,dos }}>7 \mathrm{~cm}$ as indicated in Table 4.4. Except for the uncertainty on the corrected reading of the planeparallel chamber in beam quality $Q$, additional uncertainties are two contributions from $M_{\text {corr }}$ (Table 4.2) due to the cross-calibration of the plane-parallel chamber against the cylindrical chamber in beam quality $Q_{\text {cross }}$ and a contribution for the determination of the plane-parallel chamber's $k_{Q, Q \text { cross. }}$

The expanded uncertainty for a $D_{\mathrm{w}}$ measurement ranges from $2.8 \%$ for a cylindrical chamber in the highest electron energy with $R_{50, \text { dos }}>7 \mathrm{~cm}$ (Table 4.4 ), $3.2 \%$ for a crosscalibrated plane-parallel chamber (Table 4.5 ) to $3.6 \%$ for a plane-parallel chamber calibrated in ${ }^{60} \mathrm{Co}$ (Table 4.3).

Uncertainty contributions indicated with 'audit' in Tables 4.3 to Table 4.5 indicate their contribution to the audit result as explained in the next section. 
Table 4.3: Measurement of $D_{\mathrm{w}}$ at a depth of $z_{\text {ref, }}$ based on a plane-parallel chamber directly calibrated in ${ }^{60} \mathrm{Co}$. Uncertainties indicated with 'audit' contribute to the audit result.

\begin{tabular}{|c|c|c|}
\hline source of uncertainty & & $\begin{array}{c}\text { standard } \\
\text { uncertainty } \\
/ \%\end{array}$ \\
\hline$M_{\text {corr }}:$ corrected charge measurement at $z_{\text {ref }}$ (Table 4.2) & audit & 0.37 \\
\hline$N_{D, \mathrm{w}, Q_{0}}^{P P}:$ plane-parallel chamber calibrated in ${ }^{60} \mathrm{Co}$ & & 0.50 \\
\hline$k_{Q, Q 0}:$ plane-parallel chamber quality correction (NCS 18 [1]) & & 1.70 \\
\hline uncertainty of $R_{50 \text {,dos }}$ on $k_{Q, Q 0}(0.07 \mathrm{~cm})$ & audit & 0.09 \\
\hline combined standard uncertainty $(k=1)$ & & 1.8 \\
\hline expanded uncertainty $(k=2)$ & & 3.6 \\
\hline $\begin{array}{l}\text { combined standard uncertainty contribution to audit result, based on } \\
\text { contributions indicated with 'audit' only }\end{array}$ & audit & 0.38 \\
\hline
\end{tabular}

Table 4.4: Measurement of $D_{w}$ at $R_{50 \text {,dos }}>7 \mathrm{~cm}$, depth of $z_{\text {ref, }}$ with a Farmer type cylindrical chamber calibrated in ${ }^{60} \mathrm{Co}$. Uncertainties indicated with 'audit' contribute to the audit result.

\begin{tabular}{|c|c|c|}
\hline source of uncertainty & & $\begin{array}{c}\text { standard } \\
\text { uncertainty } \\
/ \%\end{array}$ \\
\hline$M_{\text {corr }}:$ corrected charge measurement at $z_{\text {ref }} R_{50, \text { dos }}>7 \mathrm{~cm}$ (Table 4.2) & audit & 0.37 \\
\hline$N_{D, w, Q_{0}}^{C Y L}:$ Farmer type cylindrical chamber calibrated in ${ }^{60} \mathrm{Co}$ & & 0.50 \\
\hline$k_{Q, Q 0}:$ Farmer chamber beam quality correction (NCS 18 [1]) & & 1.2 \\
\hline uncertainty of $R_{50, \text { dos }}$ on cylindrical $k_{Q c r o s s, Q 0}(0.07 \mathrm{~cm})$ & audit & 0.09 \\
\hline combined standard uncertainty $(k=1)$ & & 1.4 \\
\hline expanded uncertainty $(k=2)$ & & 2.8 \\
\hline $\begin{array}{l}\text { combined standard uncertainty contribution to audit result, based } \\
\text { contributions indicated with 'audit' only }\end{array}$ & audit & 0.38 \\
\hline
\end{tabular}

Table 4.5: Measurement of $D_{\mathrm{w}}$ at a depth of $z_{\text {ref, }}$, based on a plane-parallel chamber crosscalibrated against a Farmer type cylindrical chamber at beam quality $R_{50 \text {,dos }}>7 \mathrm{~cm}$. Uncertainties indicated with 'audit' contribute to the audit result.

\begin{tabular}{|c|c|c|}
\hline source of uncertainty & & $\begin{array}{c}\text { standard } \\
\text { uncertainty } \\
/ \%\end{array}$ \\
\hline$D_{\mathrm{w}}$ at $R_{50, \mathrm{dos}}>7 \mathrm{~cm}$ with a Farmer type cylindrical chamber (Table 4.4 ) & & 1.35 \\
\hline$M_{\text {corr: }}$ corrected charge measurement at $R_{50, \text { dos }}>7 \mathrm{~cm}$ (Table 4.2 ) & audit & 0.35 \\
\hline$M_{\text {corr: }}$ corrected charge measurement at beam quality $Q$ (Table 4.2$)$ & audit & 0.35 \\
\hline$k_{Q, Q \text { cross: }}$ plane-parallel chamber quality correction (NCS 18 [1]) & & 0.60 \\
\hline influence of measurement of $R_{50, \text { dos }}$ on plane-parallel $k_{Q, Q \text { cross }}$ & audit & 0.09 \\
\hline combined standard uncertainty $(k=1)$ & & 1.6 \\
\hline expanded uncertainty $(k=2)$ & & 3.2 \\
\hline $\begin{array}{l}\text { combined standard uncertainty contribution to audit result, based on } \\
\text { contributions indicated with 'audit' only }\end{array}$ & audit & 0.50 \\
\hline
\end{tabular}




\subsection{Uncertainties in the audit results}

\subsubsection{Uncertainty in audit result for $R_{50, \text { dos, }} \Delta_{R, 50, d o s}$}

The audit result for $R_{50 \text {,dos }}$ is the difference between the participant's value and the reference value, $\Delta_{R_{50 \text {,dos }}}$ in the unit $\mathrm{cm}$ (see equation (3) section 2.1). Its standard uncertainty $u_{\Delta_{R_{50 \text {,dos }}}}$ is also expressed in the unit $\mathrm{cm}$ and is determined by the quadratic summation of the individual uncertainty contributions of the reference value and the participant's value. Since the participants didn't report uncertainties, their contributing uncertainties are assumed to be at least as high as the uncertainty of the reference value (Table 4.1), i.e. $u_{R, 50, \text { dos }}=0.07 \mathrm{~cm}$. No correlations exist between the $R_{50 \text {,dos }}$ reference value and participants value. Therefore, the combined uncertainty of the audit result, $U_{\Delta_{R_{50,0 s}}}$, is obtained by the quadratic sum of individual uncertainties (i.e. each $U=0.14 \mathrm{~cm}$ with $k=2$ ), resulting in an expanded uncertainty in the audit result:

$$
U_{\Delta, R_{50, \mathrm{dos}}}=0.20 \mathrm{~cm}
$$

\subsubsection{Uncertainty in audit result for $D_{w}, \Delta \%, D, w$}

The audit result for $D_{w}$ is the relative difference between the participant's value and the reference value, $\Delta_{D_{w}}$ in \% (see section 2.1). The uncertainty in the audit result for $D_{w}$, expressed as $u_{\Delta_{D_{w}}}$ is also expressed as a relative value and shown in Tables 4.6 until 4.8. Calibration coefficients of the dosimetry equipment of all participants and the NCS-team are traceable to the same calibration laboratory, namely VSL. In addition, the participants and NCS-team apply the same dosimetry protocol (i.e. NCS 18) which uses generic $k_{Q}$ factors for a certain ionization chamber type. Therefore, the uncertainties on the calibration coefficients and the applied $k_{\mathrm{Q}}$ factors used in the determination of $\Delta_{D_{w}}$ are partially correlated. Since $\Delta_{D_{w}}$ depends on the ratio of the calibration coefficients and the applied $k_{\mathrm{Q}}$ factors, these correlating uncertainties can be partially neglected for the determination of $U_{\Delta_{D_{w}}}$. Only the uncorrelated part of the uncertainties on the calibration coefficient and the $k_{\mathrm{Q}}$ factor are considered. This is implemented in the calculation of $U_{\Delta_{D_{w}}}$ in Table 4.5 and 4.6 in the following way. All fully uncorrelated uncertainty contributions to $U_{\Delta_{D_{W}}}$ are indicated with 'audit' in Tables 4.2 until 4.5 and are transferred to Tables 4.6 to 4.8 . For all partially correlated uncertainty contributions (calibration coefficients and $k_{\mathrm{Q}}$ ) in Tables 4.2 until 4.5, only the uncorrelated part is added to Tables 4.6 through 4.8 as long-term (<3 year) reproducibility of the 
chamber's individual calibration coefficients in ${ }^{60} \mathrm{Co}$ and $k_{Q, Q 0}$ chamber-to-chamber variation for NACP-02 and Roos type chambers respectively.

The magnitude of the plane-parallel chamber-to-chamber variation in $k_{\mathrm{Q}}$ depends on the origin of the calibration coefficient in electron beam quality $Q$, i.e. calibrated in ${ }^{60} \mathrm{Co}$ or crosscalibrated in a high-energy electron beam (described in section 3.6.2). For plane-parallel chambers calibrated in ${ }^{60} \mathrm{Co}$ the chamber-to-chamber variation is caused by a variation in $p_{\text {wall }}$ in ${ }^{60} \mathrm{Co}$ and the uncertainty related to Monte Carlo calculated perturbation corrections, $p_{Q}$ $[1,6]$. Due to a lack of information about the uncertainties responsible for chamber-tochamber variation in $k_{Q, Q 0}$ of well-guarded plane-parallel chambers, in this study an uncertainty contribution of $1.0 \%$ (see Table 4.6) is used. This uncertainty is considered to account for variations of $k_{Q, Q 0}$ between the ionization chamber of the NCS-team and that of the participants.

A similar approach is used with respect to the audit result obtained with a Farmer type cylindrical ionization chamber. However, the chamber-to-chamber variation is smaller due to a better understanding of their perturbation corrections. The uncertainty of the contributing Monte Carlo calculations is $0.77 \%$ [6] for any chamber. The uncertainty contribution as a result of the agreement in $k_{Q, Q 0}$ factors between two Farmer type ionization chambers is estimated to be within $0.50 \%$.

Tables 4.6 and 4.8 show that the audit result has an expanded uncertainty of $2.4 \%(k=2)$ regardless the method used, i.e. plane-parallel chamber calibrated in ${ }^{60} \mathrm{Co}$ or cross-calibrated in a high-energy electron beam with $R_{50, \text { dos }}>7 \mathrm{~cm}$. This is caused by the extra measurements needed to perform the cross-calibration by both institutes which cancels the advantage in smaller uncertainties for beam quality conversion factors.

Not all participants use the same method for $D_{\mathrm{w}}$ determination (some use a plane-parallel chamber calibrated in ${ }^{60} \mathrm{Co}$ others used a cross-calibrated plane-parallel chamber in a highenergy electron beam, $Q_{\text {cross }}$ ) the uncertainty of the audit result and therefore the expanded uncertainty used in the $E_{n}$-score for determination of the audit result (see section 2.2) is taken to be the uncertainty expressed for $D_{\mathrm{w}}$ measurement with plane-parallel chamber for all beam qualities at:

$$
U_{\Delta_{\%}, D_{w}}=2.4 \%
$$

Although a distinction could be made between measurements at $R_{50 \text {,dos }}>7 \mathrm{~cm}$ and $R_{50 \text {,dos }} \leq 7$ $\mathrm{cm}$, for reason of simplicity of the audit, only one value for the uncertainty is used. 
Table 4.6: The uncertainty budget for the audit results $\Delta_{D, \mathrm{w}}$ in $D_{\mathrm{w}}$ at beam quality $Q$ with a plane-parallel chamber calibrated in ${ }^{60} \mathrm{Co}$.

\begin{tabular}{lc}
\hline source of uncertainty & $\begin{array}{c}\text { standard } \\
\text { uncertainty } / \%\end{array}$ \\
\hline reference $D_{\mathrm{w}}$ value, correlations taken into account (Table 4.3) & 0.38 \\
participants $D_{\mathrm{w}}$ value, correlations taken into account (Table 4.3) & 0.38 \\
long-term (<3 year) variation of participant's $N_{D, \mathrm{w}}$ & 0.15 \\
$k_{Q, Q 0}$ individual chamber variation for plane-parallel chambers & 1.0 \\
\hline combined standard uncertainty $(k=1)$ & 1.2 \\
expanded uncertainty $(k=2)$ & 2.4 \\
\hline
\end{tabular}

Table 4.7: The uncertainty budget for the audit results $\Delta_{D, \mathrm{w}}$ in $D_{\mathrm{w}}$ at beam quality $Q$ with a Farmer type cylindrical chamber in $Q_{\text {cross }}$ with $R_{50, \text { dos }}>7 \mathrm{~cm}$.

\begin{tabular}{lc}
\hline source of uncertainty & $\begin{array}{c}\text { standard } \\
\text { uncertainty } / \%\end{array}$ \\
\hline reference $D_{\mathrm{w}}$ value at $Q_{\mathrm{cross}}$, correlations taken into account (Table 4.4) & 0.38 \\
participants $D_{\mathrm{w}}$ value at $Q_{\text {cross, correlations taken into account (Table 4.4) }}$ & 0.38 \\
long-term (<3 year) variation of participant $N_{D, \mathrm{w}}$ & 0.15 \\
$k_{Q, Q 0}$ individual chamber variation for cylindrical chambers & 0.50 \\
\hline combined standard uncertainty $(k=1)$ & 0.75 \\
expanded uncertainty $(k=2)$ & 1.5 \\
\hline
\end{tabular}

Table 4.8: The uncertainty budget for the audit results $\Delta_{D, \mathrm{w}}$ in $D_{\mathrm{w}}$ at beam quality $Q$ with a plane-parallel chamber cross-calibrated in $Q_{\text {cross }}$ with $R_{50, \text { dos }}>7 \mathrm{~cm}$.

\begin{tabular}{lc}
\hline source of uncertainty & $\begin{array}{c}\text { standard } \\
\text { uncertainty } / \%\end{array}$ \\
\hline reference $D_{\mathrm{w}}$ value at $Q_{\mathrm{cross}}$, correlations taken into account (Table 4.4) & 0.38 \\
participants $D_{\mathrm{w}}$ value at $Q_{\mathrm{cross}}$, correlations taken into account (Table 4.4) & 0.38 \\
long-term (<3 year) variation of participant $N_{D, \mathrm{w}}$ for cylindrical in ${ }^{60} \mathrm{Co}$ & 0.15 \\
$k_{Q, Q 0}$ individual chamber variation for cylindrical chambers & 0.50 \\
\hline reference $D_{\mathrm{w}}$ value at $Q$, correlations taken into account (Table 4.5) & 0.50 \\
participants $D_{\mathrm{w}}$ value at $Q$, correlations taken into account (Table 4.5) & 0.50 \\
$k_{Q, Q 0}$ individual chamber variation for plane-parallel chambers & 0.50 \\
\hline combined standard uncertainty $(k=1)$ & 1.2 \\
expanded uncertainty $(k=2)$ & 2.4 \\
\hline
\end{tabular}




\section{Results}

\subsection{Reference and participants' values}

Table 5.1 gives the reference and participant's values for $R_{50, \text { dos }}$ and $D_{\mathrm{w}}$ during the audit measurements at the participants sites. For completeness the reference depth, $z_{\text {ref, }}$, is reported. The $D_{\mathrm{w}}$ reference value is based on measurement with the plane-parallel chamber both calibrated in ${ }^{60} \mathrm{Co}$ (column indicated with $D_{\mathrm{w}}$ ) according to 3.6 .1 and based on a crosscalibration in a high-energy electron beam (column indicated with $D_{\mathrm{w}}{ }^{* *}$ ) according to section 3.6.2. The uncertainties are $3.6 \%$ and $3.2 \%(k=2)$, as respectively reported in table 4.3 and table 4.5 of section 4.2 .

Table 5.1: Reference values and participants' values during the audit. Values indicated with ** are based on a cross-calibration of a plane-parallel chamber in a high-energy electron beam.

\begin{tabular}{|c|c|c|c|c|c|c|c|c|c|}
\hline \multirow{2}{*}{$\begin{array}{c}\text { site } \\
\text { (see } \\
\text { Table 1.1) }\end{array}$} & \multirow{2}{*}{$\begin{array}{c}\text { date } \\
(2015)\end{array}$} & \multirow{2}{*}{$\begin{array}{c}E \\
/ \mathrm{MeV}\end{array}$} & \multicolumn{4}{|c|}{ reference value } & \multicolumn{3}{|c|}{ participants' value } \\
\hline & & & $\begin{array}{l}R_{50, \mathrm{dos}} \\
\quad / \mathrm{cm}\end{array}$ & $\begin{array}{l}Z_{\mathrm{ref}} \\
/ \mathrm{cm}\end{array}$ & $\begin{array}{c}D_{\mathrm{w}} \\
\text { /cGy }\end{array}$ & $\begin{array}{l}D_{w}^{* *} \\
/ c G y\end{array}$ & $\begin{array}{l}R_{50, \mathrm{dos}} \\
/ \mathrm{cm}\end{array}$ & $\begin{array}{l}z_{\mathrm{ref}} \\
/ \mathrm{cm}\end{array}$ & $\begin{array}{c}D_{\mathrm{w}} \\
\text { /cGy }\end{array}$ \\
\hline & & 6 & 2.54 & 1.42 & 199.4 & 197.6 & 2.63 & 1.48 & $198.4^{\star \star}$ \\
\hline \multirow[t]{3}{*}{ AVL } & $16 / 07$ & 12 & 4.88 & 2.83 & 200.8 & 198.9 & 4.99 & 2.89 & $199.7^{\star *}$ \\
\hline & & 18 & 7.20 & 4.22 & 197.1 & 195.3 & 7.35 & 4.31 & $196.1^{* *}$ \\
\hline & & 4 & 1.62 & 0.87 & 197.1 & - & 1.60 & 0.86 & 197.3 \\
\hline \multirow[t]{3}{*}{$\mathrm{RdG}$} & $30 / 07$ & 10 & 3.99 & 2.29 & 196.0 & - & 4.00 & 2.30 & 196.8 \\
\hline & & 15 & 6.00 & 3.50 & 196.0 & - & 6.27 & 3.66 & 195.6 \\
\hline & & 6 & 2.39 & 1.34 & 304.8 & 305.8 & 2.38 & 1.33 & $304.8^{\star *}$ \\
\hline \multirow[t]{3}{*}{ VUmc } & $25 / 08$ & 9 & 3.63 & 2.08 & 296.1 & 297.0 & 3.60 & 2.06 & $296.9^{* \star}$ \\
\hline & & 22 & 8.75 & 5.15 & 286.5 & 287.4 & 8.78 & 5.17 & $286.8^{* *}$ \\
\hline & & 4 & 1.61 & 0.86 & 199.3 & - & 1.60 & 0.86 & 199.6 \\
\hline \multirow[t]{3}{*}{ LUMC } & $15 / 09$ & $4 \mathrm{HDRE}^{*}$ & 1.58 & 0.85 & 200.9 & - & 1.60 & 0.86 & 202.7 \\
\hline & & 12 & 4.64 & 2.69 & 200.0 & - & 4.78 & 2.77 & 199.3 \\
\hline & & 6 & 2.55 & 1.43 & 199.6 & 199.4 & 2.51 & 1.41 & $198.7^{* *}$ \\
\hline \multirow[t]{2}{*}{$A V L$} & $05 / 11$ & 12 & 4.93 & 2.86 & 198.8 & 198.6 & 4.95 & 2.87 & $197.4^{\star \star}$ \\
\hline & & 18 & 7.22 & 4.24 & 197.4 & 197.2 & 7.32 & 4.29 & $195.4^{\star \star}$ \\
\hline
\end{tabular}

\footnotetext{
* High Dose Rate Electron beam (HDRE) with the application of NCS 18 [1] at a field size of $40 \times 40 \mathrm{~cm}^{2}$.

**Value(s) obtained via a cross-calibration of a plane-parallel chamber against a farmer chamber in a high-energy electron beam according to section 3.6.2.
}

\subsection{Audit results}

Table 5.2 and Figures 5.1 and 5.2 give the audit results for the participants in this study. Audit results are based on a reference value with the plane-parallel chamber both calibrated 
in ${ }^{60} \mathrm{Co}$ (column indicated with $\Delta_{D_{\mathrm{w}}}$ ), see section 3.6.1, and based on a cross-calibration in a


uncertainties on both audit results are both $2.4 \%(k=2)$ as respectively reported in Table 4.6 and Table 4.8 of section 4.2 .

The audit results indicated 'satisfactory' or 'unsatisfactory' in the final column are based on the analyses of the $E_{n}$-scores. $E_{n}$-scores for $D_{w}$ measurement range between 0.0 and 0.6 show excellent agreement. Based on these $E_{n}$-scores at all 15 beam qualities between $4 \mathrm{MeV}$ and $22 \mathrm{MeV}$, it can be concluded that the audit results for the $D_{\mathrm{w}}$ measurements of all institutes were 'satisfactory'.

Table 5.2 and the Figures 5.1 and 5.2 also show that only one of the $E_{n}$-scores for $R_{50 \text {,dos }}$ is $>1$. All other 14 audit measurements for $R_{50, \text { dos }}$ resulted in a satisfactory $E_{n}$-score. It should be noted that despite the fact that one of the results for $R_{50 \text {,dos }}$ might be qualified as 'unsatisfactory', it's value is within $k=3$ (99\% coverage interval and corresponding to $0.21 \mathrm{~cm}$ ) from the reference value.

The audit results (Figure 5.1 and Table 5.2) show a noticeable difference of about $1 \%$ between the ${ }^{60} \mathrm{Co}$ and cross-calibrated reference values during the first audit (AVL 16/07). This difference is not present during the final audit (AVL 05/11), conducted at the same institute. The difference between ${ }^{60} \mathrm{Co}$ and cross-calibrated values are about $0.1 \%$ instead. Additionally, both audits show equal $\mathrm{E}_{\mathrm{n}}$-scores on the audit result. 
Table 5.2: Audit results for $R_{50, \text { dos }}$ and $D_{\mathrm{w}}$ in 15 electron beams at the 4 participating institutes of this study.

\begin{tabular}{|c|c|c|c|c|c|c|c|c|c|}
\hline \multirow[b]{2}{*}{$\begin{array}{c}\text { Site } \\
\text { (see } \\
\text { Table 1.1) }\end{array}$} & \multirow[b]{2}{*}{$\begin{array}{c}\text { date } \\
(2015)\end{array}$} & \multirow[b]{2}{*}{$\begin{array}{c}E \\
/ \mathrm{MeV}\end{array}$} & \multicolumn{2}{|c|}{$R_{50 \text {,dos }}$} & \multicolumn{5}{|c|}{$D_{\mathrm{w}}$} \\
\hline & & & $\begin{array}{c}\Delta_{R_{50, \mathrm{dos}}} \\
/ \mathrm{cm}\end{array}$ & $\begin{array}{c}E_{n^{-}} \\
\text {score }\end{array}$ & $\begin{array}{c}\Delta_{D_{\mathrm{w}}} \\
/ \%\end{array}$ & $\begin{array}{c}E_{n^{-}} \\
\text {score }\end{array}$ & $\begin{array}{c}\Delta_{D_{\mathrm{w}}{ }^{* *}} \\
1 \%\end{array}$ & $\begin{array}{c}E_{n^{-}} \\
\text {score }\end{array}$ & PT result \\
\hline & & 6 & 0.09 & 0.5 & -0.52 & 0.2 & 0.40 & 0.2 & satisfactory \\
\hline \multirow[t]{3}{*}{$A V L$} & $16 / 07$ & 12 & 0.11 & 0.6 & -0.51 & 0.2 & 0.42 & 0.2 & satisfactory \\
\hline & & 18 & 0.15 & 0.8 & -0.55 & 0.2 & 0.38 & 0.2 & satisfactory \\
\hline & & 4 & -0.02 & 0.1 & 0.09 & 0.0 & - & $=$ & satisfactory \\
\hline \multirow[t]{3}{*}{$\mathrm{RdG}$} & $30 / 07$ & 10 & 0.01 & 0.1 & 0.42 & 0.2 & - & - & satisfactory \\
\hline & & 15 & 0.27 & 1.4 & -0.20 & 0.1 & - & - & satisfactory \\
\hline & & 6 & -0.01 & 0.1 & 0.02 & 0.0 & -0.30 & 0.1 & satisfactory \\
\hline \multirow[t]{3}{*}{ VUmc } & $25 / 08$ & 9 & -0.03 & 0.2 & 0.28 & 0.2 & -0.04 & 0.0 & satisfactory \\
\hline & & 22 & 0.03 & 0.2 & 0.08 & 0.0 & -0.23 & 0.1 & satisfactory \\
\hline & & 4 & -0.01 & 0.1 & 0.14 & 0.1 & - & - & satisfactory \\
\hline \multirow[t]{3}{*}{ LUMC } & $15 / 09$ & 4 HDRE* $^{*}$ & 0.02 & 0.1 & 0.90 & 0.4 & - & - & satisfactory \\
\hline & & 12 & 0.14 & 0.7 & -0.39 & 0.2 & - & - & satisfactory \\
\hline & & 6 & -0.03 & 0.2 & -0.43 & 0.2 & -0.34 & 0.1 & satisfactory \\
\hline \multirow[t]{2}{*}{$A V L$} & $05 / 11$ & 12 & 0.02 & 0.1 & -0.69 & 0.3 & -0.59 & 0.3 & satisfactory \\
\hline & & 18 & 0.10 & 0.5 & -1.0 & 0.4 & -0.94 & 0.6 & satisfactory \\
\hline
\end{tabular}

\footnotetext{
* High Dose Rate Electron beam (HDRE) with the application of NCS 18 [1] at a field size of $42 \times 42 \mathrm{~cm}^{2}$, which is larger than the surface of the audit phantom,

${ }^{\star *}$ Value(s) obtained via a cross-calibration of a plane-parallel chamber against a farmer chamber in a high-energy electron beam according to section 3.6.2.
} 




Figure 5.1: Audit results for $R_{50, \text { dos }}$ in the 15 electron beams of the 4 participants. The dotted line through $\Delta_{D, \mathrm{w}}=$ $0.0 \%$ represents the reference value. Circles and crosses respectively indicate reference values based on a plane-parallel chamber calibrated in ${ }^{60} \mathrm{Co}$ and cross-calibrated in a high-energy electron beam.

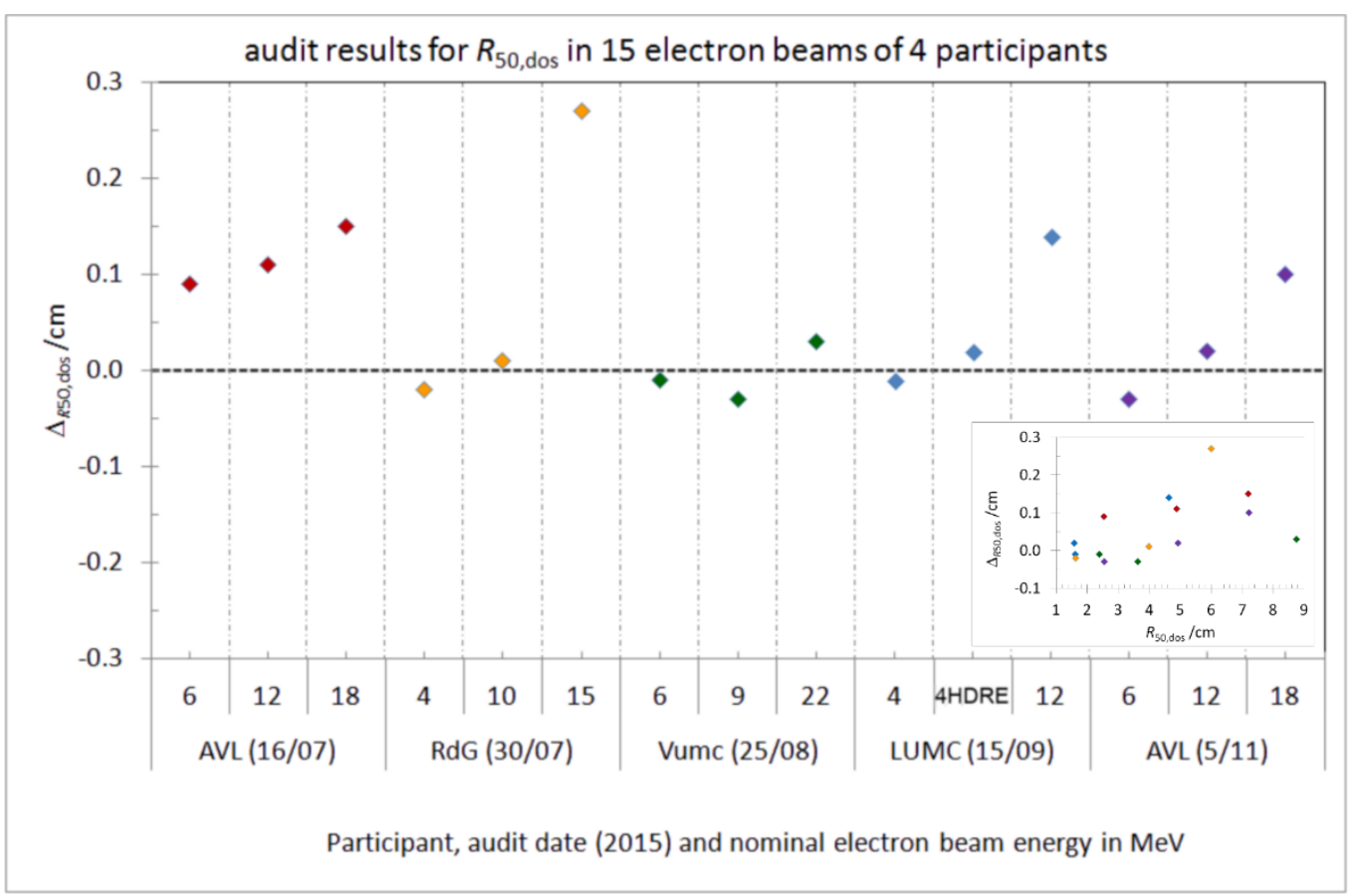

Figure 5.2: Audit results for $D_{\mathrm{w}}$ in the 15 electron beams of the 4 participants and as a function of $R_{50 \text {,dos }}$ (bottomright inlay). 


\section{Discussion and conclusion}

During this study, a total of 15 electron beams were measured in 4 participating institutes. One institute was measured twice, i.e. at the beginning of the audit campaign as an initial test audit and at the end of the audit campaign as an actual audit. Both results have been reported.


electron beam with an unsatisfactory $E_{n}$-score of 1.4 . Besides that, this value still agreed within 3 standard uncertainties, it did not influence the audit result on the $D_{\mathrm{w}}$ measurement and therefore the overall audit result of this participant.

All $D_{\mathrm{w}}$ audit results, $\Delta_{D \mathrm{w}}$, turned out to be satisfactory (i.e. $\left|E_{n}\right| \leq 1.0$ ) regardless the choice of calibration method for the plane-parallel chamber (i.e. ${ }^{60} \mathrm{Co}$-calibrated or cross-calibrated in a high-energy electron beam). However, at the first (test) audit a discrepancy of $1 \%$ was found between $D_{\mathrm{w}}$ values based on the ${ }^{60} \mathrm{Co}$-calibration compared to its cross-calibration based value. Since the ${ }^{60} \mathrm{Co}$-based value of the first audit was consistent with both ${ }^{60} \mathrm{Co}$-based and cross-calibration based values of the final audit at the same department, it was concluded that the cross-calibration of the NCS audit team at the first (test) audit was incorrect. The error is expected to be caused by the lack of experience in use of the NCS-equipment; more specifically misalignment of the Farmer type cylindrical chamber at the reference depth. This was confirmed by the ${ }^{60} \mathrm{Co}$-based value at the beam quality which was consistent with the value measured at the final audit.

With respect to the uncertainty for the determination of absorbed dose under reference conditions, the smallest uncertainty of $2.8 \%$ is achieved with a Farmer type cylindrical chamber in a high-energy electron beam with $R_{50, \text { dos }}>7 \mathrm{~cm}$ (Table 4.4). Generally, for all electron beam qualities, the smallest uncertainty of $3.2 \%$ is achieved with a cross-calibrated plane-parallel chamber against a Farmer chamber in a high-energy electron beam when this calibration is performed with care (Table 4.5). The third option, however a much simpler and time efficient measurement, is to obtain $D_{\mathrm{w}}$ with a plane-parallel chamber calibrated in ${ }^{60} \mathrm{Co}$ and results in an uncertainty of $3.6 \%$ (Table 4.3).

In this study the uncertainty on the audit result based on a ${ }^{60} \mathrm{Co}$ calibrated plane-parallel chamber or a cross-calibrated plane-parallel chamber was estimated to be equal, i.e. $2.4 \%$, when care is taken performing the cross-calibration (Table 4.6 and Table 4.8). Although a distinction could be made for the audit results above $R_{50 \text {,dos }}>7 \mathrm{~cm}$ with a smaller standard uncertainty of $1.5 \%$ (Table 4.7 ) for reason of simplicity of the audit, a single uncertainty value of $2.4 \%$ was used. 
It should be noted that the audit described in this study, was performed at radiotherapy departments that are using measurement instruments traceable to VSL and that are applying the same code of practice as the NCS audit team. If this audit would be performed at a department that uses measurement equipment not traceable to VSL or applies a different dosimetry protocol than the NCS audit team, the uncertainties reported in this study would not apply.

Overall it can be concluded that the audit has been implemented successfully. Differences of $D_{\mathrm{w}}$ values between the audit team and the participant were in all cases smaller than $1 \%$ and in most cases smaller than $0.5 \%$ with a relative uncertainty of $2.4 \%(k=2)$. Differences in $R_{50 \text {,dos }}$ were in all cases smaller than $0.3 \mathrm{~cm}$ and in most cases smaller than $0.2 \mathrm{~cm}$ with an uncertainty of $0.20 \mathrm{~cm}$. Except for the first (test) audit, $D_{\mathrm{w}}$ values obtained with a planeparallel chamber calibrated in ${ }^{60} \mathrm{Co}$ agreed within $0.3 \%$ with $D_{\mathrm{w}}$ values obtained with plane parallel chambers cross-calibrated in a high-energy electron cross-calibration.

\section{Acknowledgements}

The subcommittee would like to thank Wenze van Klink for his work for the subcommittee as member during his employment at the VU Medical Center. 


\section{References}

[1] Aalbers AHL, Hoornaert M-T, Minken A, Palmans H, Pieksma MWH, De Prez LA, Reynaert N, Vynckier S, Wittkämper FW, NCS Report 18: Code of practice for the absorbed dose determination in high energy photon and electron beams, Delft, 2008. doi:10.25030/ncs-018.

[2] Perik TJ, De Prez LA, Aalbers AHL, Monseux A, Sergent F, Wittkämper FW, Van de Kamer JB, NCS Report 23: Audit of high-energy photon beams in Belgian and Dutch radiotherapy departments, Delft, 2013. doi:10.25030/ncs-023.

[3] ISO/IEC, Conformity assessment - General requirements for proficiency testing (ISO/IEC 17043:2010, IDT), Geneva, 2010.

[4] Mijnheer BJ, Aalbers AHL, Van Gasteren JJM, Visser AG, Wittkämper FW, NCS Report 2: Code of practice for the dosimetry of high-energy photon beams, Delft, 1986. doi:10.25030/ncs-002.

[5] Wittkämper FW, Aalbers AHL, Brouwer WFM, Huizenga H, Mijnheer BJ, Piron AJ, Vynckier S, NCS Report 5: Code of practice for the dosimetry of high-energy electron beams, Delft, 1989. doi:10.25030/ncs-005.

[6] Andreo P, Burns DT, Hohlfield K, Huq MS, Kanai T, Laitano F, Smyth VG, Vynckier S, IAEA TRS-398: Absorbed Dose Determination in External Beam Radiotherapy, International Atomic Energy Agency, Vienna, 2006.

[7] Thwaites DI, Burns DT, Klevenhagen SC, Nahum AE, Pitchford WG, The IPEMB code of practice for electron dosimetry for radiotherapy beams of initial energy from 2 to 50 MeV based on an air kerma calibration, Phys. Med. Biol. 41 (1996) 2557-2603. doi:10.1088/0031-9155/41/12/001.

[8] Zink K, Czarnecki D, Looe HK, von Voigts-Rhetz P, Harder D, Monte Carlo study of the depth-dependent fluence perturbation in parallel-plate ionization chambers in electron beams, Med. Phys. 41 (2014) 111707. doi:10.1118/1.4897389.

[9] Weinhous MS, Meli JA, Determining Pion, the correction factor for recombination losses in an ionization chamber., Med. Phys. 11 (1984) 846-849. doi:10.1118/1.595574.

[10] Bichsel H, Pierson DH, Boring JW, Green A, Inokuti M, Hurst G, ICRU report 31: Average Energy Required to Produce an Ion Pair, Washington D.C., 1979. doi:10.1017/CBO9781107415324.004.

[11] JCGM, Evaluation of measurement data: Guide to the expression of uncertainty in measurement, Bureau International des Poids et Mesures, 2008. doi:10.1373/clinchem.2003.030528. 
http://www.doi.org/10.25030/ncs-029 This NCS report has been downloaded on 26 Apr 2023 